\title{
Erratum: Anisotropic-Exchange Magnets on a Triangular Lattice: Spin Waves, Accidental Degeneracies, and Dual Spin Liquids [Phys. Rev. X 9, 021017 (2019)]
}

\author{
P. A. Maksimov, Zhenyue Zhu, Steven R. White, and A. L. Chernyshev $\odot$
}

(Q) (Received 14 December 2021; published 16 February 2022)

DOI: 10.1103/PhysRevX.12.019902

It was brought to our attention that the transformation matrix from the cubic to crystallographic reference frame, $\mathbf{S}_{\text {cryst }}=\hat{\mathbf{R}}_{c} \mathbf{S}_{\text {cubic }}$, Eq. (43) of our work, used an unconventional orientation of the cubic axes triad, corresponding to $\mathrm{x} \rightarrow-\mathrm{x}$ and $\mathrm{y} \rightarrow-\mathrm{y}$ of the conventional choice for the cubic reference frame. While presumably leading to the same physical results, our transformation should have resulted in a model that is different from the extended Kitaev-Heisenberg model listed in Eq. (44) by more complicated sign conventions for the $\Gamma$ and $\Gamma^{\prime}$ terms on different bonds.

To adhere to the conventional and symmetric choice, the transformation matrix $\hat{\mathbf{R}}_{c}$ in Eq. (43) should read as

$$
\hat{\mathbf{R}}_{c}=\left(\begin{array}{ccc}
0 & -\frac{1}{\sqrt{2}} & \frac{1}{\sqrt{2}} \\
\sqrt{\frac{2}{3}} & -\frac{1}{\sqrt{6}} & -\frac{1}{\sqrt{6}} \\
\frac{1}{\sqrt{3}} & \frac{1}{\sqrt{3}} & \frac{1}{\sqrt{3}}
\end{array}\right),
$$

corresponding to the conventional choice of the cubic axes shown in the modified Fig. 11.

The relation between parameters of the extended $K-J$ model [Eq. (44)] and that of the original model written in the crystallographic reference frame [Eq. (7)], which was provided by our Eq. (45), was not affected by this unconventional choice up to a nearly inconsequential change of sign in the $J_{z \pm}$ term. As we discuss throughout the paper, the sign of the $J_{z \pm}$ term leaves all eigenvalues intact for a rather transparent physical reason. However, it may affect a convention for the spin directions in the ordered state. To be consistent, we correct the sign in the $J_{z \pm}$ term below:

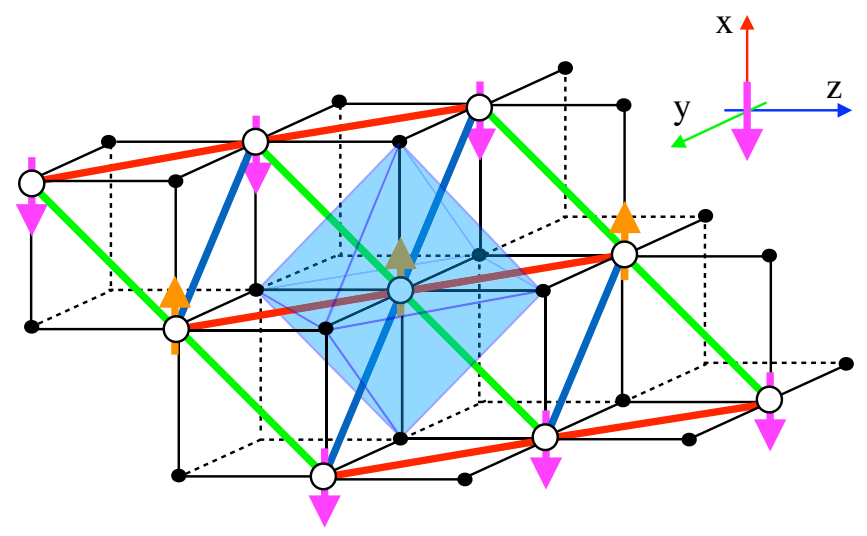

FIG. 11. Same as Fig. 1, side view. The octahedron of ligands is highlighted; cubic axes and bonds of the triangular lattice are shown. Spin ordering corresponds to the stripe-yz phase for the parameters along the gapless $K-J$ line (see text).

Published by the American Physical Society under the terms of the Creative Commons Attribution 4.0 International license. Further distribution of this work must maintain attribution to the author(s) and the published articles title, journal citation, and DOI. 


$$
\begin{aligned}
J_{0} & =\frac{1}{3}\left(2 J+\Delta J+2 J_{ \pm \pm}-\sqrt{2} J_{z \pm}\right), \\
K & =-2 J_{ \pm \pm}+\sqrt{2} J_{z \pm}, \\
\Gamma & =\frac{1}{3}\left(-J+\Delta J-4 J_{ \pm \pm}-\sqrt{2} J_{z \pm}\right), \\
\Gamma^{\prime} & =\frac{1}{6}\left(-2 J+2 \Delta J+4 J_{ \pm \pm}+\sqrt{2} J_{z \pm}\right) .
\end{aligned}
$$

These unfortunate oversights were secondary to our discussion and did not affect results presented in our work.

We are thankful to David Kaib for pointing out these inconsistencies. 